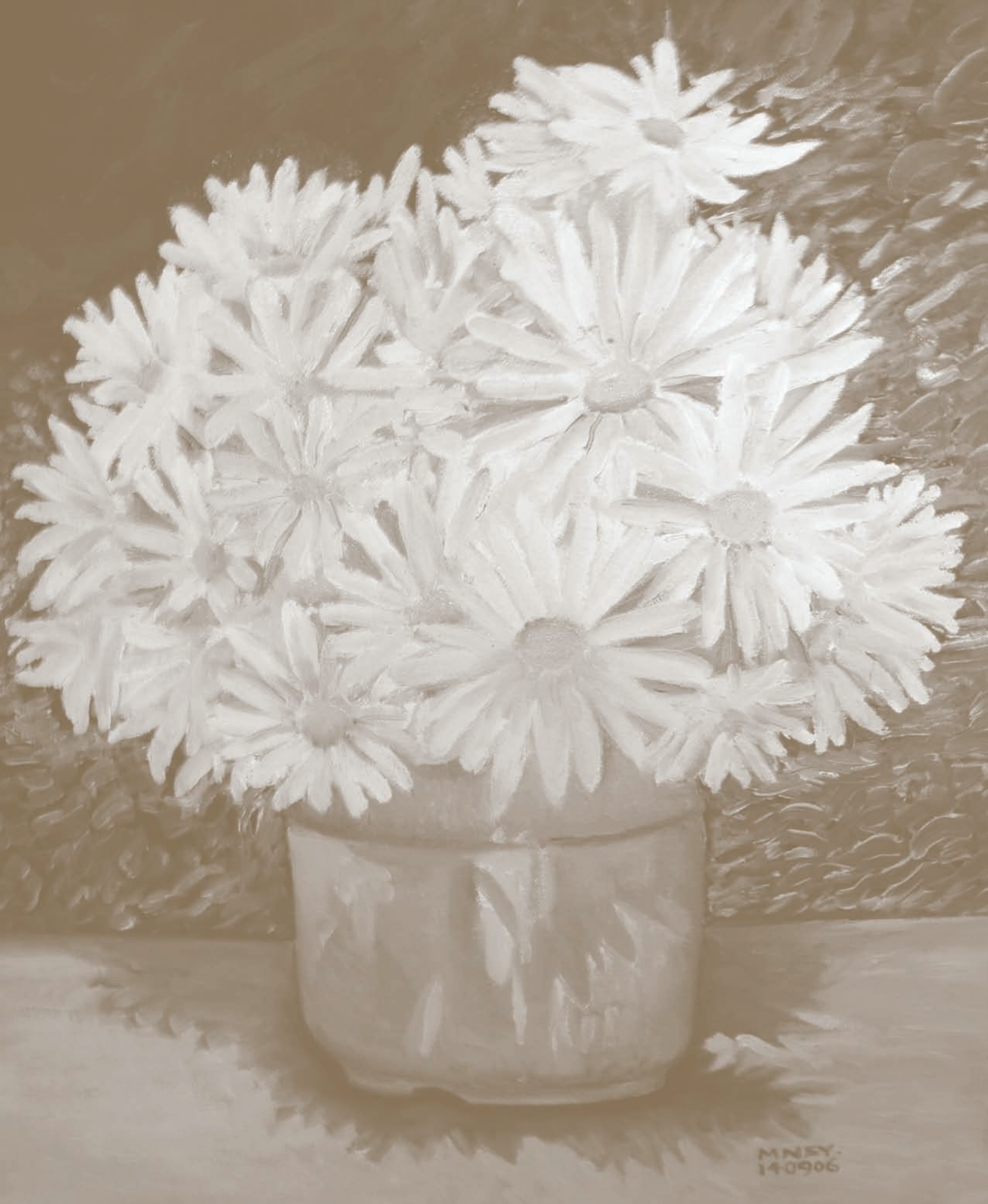




\section{Cuba: envejecimiento de la población y estado conyugal}

Raúl Hernández Castellón

\section{INTRODUCCIÓN}

Al ser las personas más longevas, cada vez durarán más tiempo y por tanto esto tendrá sus consecuencias para las relaciones familiares. Esto asegura la posibilidad de criar a los hijos en compañía de la pareja. Como, además, cada vez es menos probable que alguno de los hijos muera antes de que sus progenitores alcancen la madurez, todo esto reduce el componente de incertidumbre a la hora de planificar el futuro, pero también cambia el significado del matrimonio, ya que fácilmente puede durar más de cincuenta años y la crianza de los hijos sólo ocupará una parte de estos años.

De este modo, el envejecimiento de la población se relaciona también con el futuro de la familia. Con mucha frecuencia se ha hablado de la crisis de la familia como institución, debido entre otras causas a los avances en la liberación de la mujer y la aparición de nuevas formas de convivencia no familiares. En el fondo, se aduce que el crecimiento de la economía de mercado, de la industrialización y del Estado, ha restado a la familia sus funciones tradicionales, vaciándola de contenido y dejándole sólo las funciones afectivas y reproductivas, para las cuales es suficiente la familia nuclear. La evidencia muestra que los hogares nucleares son cada vez una mayor proporción del total, lo que demostraría la veracidad de esta teoría.

También se puede analizar el envejecimiento en función de los cambios en las relaciones entre hombres y mujeres. Una posibilidad es que el sexo deje de ser el principal determinante a la hora de asignar las funciones de cada cuál, y que en el futuro, ese factor sea la edad. En este sentido se mantienen la función productiva de los hombres jóvenes y la función reproductiva de las muje- res que han alcanzado la madurez, pero aparecen como emergentes, la función productiva de las mujeres jóvenes y las funciones familiares de los hombres maduros.

Hay que destacar, por otro lado, la "feminidad" de las edades maduras. Las mujeres son más longevas, por lo tanto, son mayoría entre los adultos mayores, y a medida que se avanza en la edad, esta diferencia se amplía. Una consecuencia es la mayor viudez femenina, que se agrava aún más por el hecho de que tradicionalmente la edad de los hombres al matrimonio era mayor. A primera vista, parecería que la situación de estas mujeres es bastante penosa. A los estigmas propios de la edad, hay que añadir sus menores recursos educativos y económicos en relación con los hombres. No obstante, el hecho de que su especialización hasta entonces haya sido la reproducción, las capacita más para las condiciones de vida propias de la tercera edad que a los que han dedicado su vida activa a las actividades productivas, ya que las habilidades adquiridas durante esta etapa ya no tienen aplicación en su nueva vida como jubilados. Por lo tanto, la jubilación difumina las diferencias entre hombres y mujeres, ya que los roles tradicionales femeninos proporcionan a la mujer madura una independencia y una autosuficiencia de la que carecen los hombres de la misma edad.

En América Latina y el Caribe se ha debatido ampliamente sobre esta temática, la que se vincula muy estrechamente con aspectos del desarrollo económico y social. Asimismo, se ha discutido mucho sobre cuáles son las principales características de su comportamiento. Resulta necesario realizar un análisis de estos aspectos en el país a través de las últimas décadas, así como sus diferencias según distintos atributos de la población y también los cambios de sus roles dentro del hogar, es decir, 
la dinámica del lugar que ocupan dentro del hogar, con relación al jefe del núcleo. Los aspectos antes señalados son el objeto de esta investigación. Este trabajo pretende más bien ser un pequeño punto de partida para la realización de estudios más profundos, que contribuyan a dilucidar los complejos problemas que abarca.

\section{DINÁMICA DEL ESTADO CONYUGAL DE LA PO- BLACIÓN DE 60 AÑOS Y MÁS EN CUBA.}

\section{I.1 Un análisis global.}

Los atributos edad y sexo de la población muestran su influencia en los cambios del estado conyugal en los últimos cincuenta años. En principio resulta interesante apreciar cuál es la variabilidad del mismo cuando intervienen el sexo, la edad y el tiempo. No obstante, antes de entrar en el análisis de las edades, conviene en primer término echarle un vistazo al comportamiento del estado conyugal de las poblaciones de 15 años y más, e incluso la de 60 años y más, por sexo.

Con relación a la población de 15 a 59 años, el aumento en la proporción de personas casadas y unidas que se muestra entre los años 1953 y 1981 pudiera quizás deberse a la campaña de legalización de uniones consensuales llevada a cabo en los primeros años de la Revolución. En ambos sexos los cambios con relación al 2002 son poco importantes. Cabe también resaltar una diferencia relativamente importante en dichas proporciones por sexo, con valores superiores para las mujeres, lo cual podría atribuirse a la tendencia de las mujeres a tener una mejor declaración de la unión conyugal.

Por su parte, las diferencias en el estado conyugal antes mencionado son mucho más importantes entre los adultos mayores por sexo. En efecto, en los tres años considerados las diferencias superan los 22 puntos porcentuales, con valores superiores en los hombres. Aquí, aparte de lo señalado como posible causa en el párrafo anterior, pudiera estar influyendo el hecho de la mayor propensión masculina a contraer nuevas nupcias al perder el vínculo conyugal por cualquier causa, es decir, viudez, divorcio o separación.
La transición demográfica cubana parece haber jugado su papel en todos estos cambios, por cuanto fue en 1978 cuando se alcanzó una fecundidad por debajo del nivel de reemplazo, como resultado de una fuerte reducción de la misma. Así, los niveles de soltería han ido disminuyendo en cada uno de los sexos, de forma más importante para toda la población, es decir, el grupo de 15 a 59 años, en particular entre 1953 y 1981.

Cabe reflexionar que no se puede atribuir este comportamiento solamente a un aumento de las proporciones de casados y unidos, pues el mismo fue discreto. Podría tratarse de una combinación, pues la disminución en diez puntos porcentuales en estas proporciones se podría compensar quizás con el incremento de las proporciones de divorciados y el de casados y unidos. En resumen: este aspecto requiere estudiarse de manera más profunda.

\section{Cuadro No. 1. Cuba: Estado conyugal de la población de 15-59 y de 60 y + años. Porcentajes. 1953, 1981 y 2002.}

\begin{tabular}{|c|c|c|c|c|}
\hline \multirow[b]{2}{*}{1953} & \multicolumn{2}{|c|}{ Hombres } & \multicolumn{2}{|c|}{ Mujeres } \\
\hline & $15-59$ & $60 y+$ & $15-59$ & $60 y+$ \\
\hline Casados/Unidos & 52,6 & 67,9 & 62,2 & 42,4 \\
\hline Solteros & 45,8 & 16,4 & 32,5 & 11,6 \\
\hline $\begin{array}{l}\text { Divorciados/ } \\
\text { Separados }\end{array}$ & 0,7 & 0,9 & 1,7 & 1,3 \\
\hline \multirow[t]{2}{*}{ Viudos } & 0,9 & 14,8 & 3,6 & 44,7 \\
\hline & 100 & 100 & 100 & 100 \\
\hline \multicolumn{5}{|l|}{1981} \\
\hline Casados/Unidos & 57,3 & 68,5 & 64,0 & 46,0 \\
\hline Solteros & 36,2 & 12,6 & 21,4 & 8,2 \\
\hline $\begin{array}{l}\text { Divorciados/ } \\
\text { Separados }\end{array}$ & 6 & 8,1 & 12,6 & 9,9 \\
\hline \multirow[t]{2}{*}{ Viudos } & 0,5 & 10,8 & 2,0 & 35,9 \\
\hline & 100 & 100 & 100 & 100 \\
\hline \multicolumn{5}{|l|}{2002} \\
\hline Casados/Unidos & 59,0 & 67,3 & 64,6 & 44,4 \\
\hline Solteros & 33,5 & 11,5 & 20,8 & 8,6 \\
\hline $\begin{array}{l}\text { Divorciados/ } \\
\text { Separados }\end{array}$ & 7,1 & 10,1 & 12,8 & 13,1 \\
\hline \multirow[t]{2}{*}{ Viudos } & 0,4 & 11,1 & 1,8 & 33,9 \\
\hline & 100 & 100 & 100 & 100 \\
\hline
\end{tabular}

Fuente: Elaborado a partir de la información de los censos respectivos. 
Quizás una manera de resumir esta discusión sea la de observar cómo se comporta el índice de masculinidad de la población de 15 a 59 años, en comparación con la de 60 años y más. En general, podría decirse, después de llevar a cabo una revisión de la información que brinda el siguiente cuadro, que se confirman los planteamientos que se han hecho en las páginas anteriores.

En efecto, los índices de masculinidad de la población adulta mayor muestran una tendencia decreciente en todos los estados conyugales, como resultado, por una parte, de la reducción de la mortalidad y por otra de la mayor sobrevivencia femenina.

Otro hecho interesante es el de la reducción en la proporción de solteros después de 1970, así como el gran incremento en los valores de los divorciados. Si se analizan estos estados conyugales según la zona de residencia, quizás podrían encontrarse algunas posibles causas de este comportamiento. En efecto, se puede apreciar que aunque los hombres urbanos disminuyeron unos 5 puntos porcentuales en sus proporciones de solteros, sin embargo, los residentes en las áreas rurales lo hicieron en algo más de 9 puntos porcentuales. Por otra parte, en el total de ambas zonas se observaron algunos cambios en las proporciones de divorciados, que son relativamente intensos en ambas zonas y en ambos sexos. Este es un aspecto que requiere de un mayor estudio, para poder elaborar las hipótesis sobre las causas de estos importantes cambios. No se incluye el año de 1953, porque en el mismo no se publicó esta información por zonas de residencia. aunque en el caso de las viudas, particularmente, ello se vincula con su mayor nivel de mortalidad. En general, si se tiene presente que Cuba y Uruguay son los dos países de mayor avance en su transición demográfica, podría asumirse como una explicación factible. Brasil y México se encuentran en un nivel inferior de avance de la citada transición.

Los hombres cubanos muestran una menor proporción de casados/unidos que los otros tres países. Su valor está más cercano al de Uruguay, posiblemente debido a que ambos están en la misma etapa de la transición demográfica, que es la más avanzada de América Latina y eso posiblemente influye en el hecho de presentar las más altas proporciones de adultos mayores. Cabría quizás avanzarse la hipótesis de que este mayor desarrollo demográfico pueda propender a mantener menos lazos matrimoniales o uniones.

Cuadro No. 2. Cuba: Índice de masculinidad de la población de 15 a 59 años y de 60 años y de $60+$ años, según estado conyugal. 1953, 1981, 2002

\begin{tabular}{|l|r|r|r|r|r|r|}
\hline & \multicolumn{3}{|c|}{$15-59$} & \multicolumn{3}{c|}{$60+$} \\
\hline & 1953 & 1981 & 2002 & 1953 & 1981 & 2002 \\
\hline $\begin{array}{l}\text { Casados/ } \\
\text { Unidos }\end{array}$ & 88,3 & 90,0 & 91,8 & 184,2 & 154,6 & 139,4 \\
\hline Solteros & 147,1 & 170,4 & 162,0 & 163,1 & 158,3 & 123,1 \\
\hline $\begin{array}{l}\text { Divorciados/ } \\
\text { Separados }\end{array}$ & 38,9 & 48,0 & 55,8 & 77,1 & 84,0 & 70,5 \\
\hline Viudos & 26,5 & 22,4 & 23,4 & 38,1 & 38,5 & 30,3 \\
\hline Total & 104,4 & 100,5 & 99,0 & 115,0 & 103,8 & 92,0 \\
\hline
\end{tabular}

Fuente: Elaborado a partir de la información de los censos respectivos.
Cabría preguntarse: ¿Cómo se comporta el estado conyugal en algunos países de América Latina en fechas alrededor del año 2000?.

Brasil es el país de mayores proporciones de casados y de viudas entre los 13 países en los que se recopiló información en un trabajo publicado hace pocos años. ${ }^{1}$ Los patrones culturales prevalecientes podrían estar asociados con este comportamiento, por una parte,
Cuadro 3. Cuba: Estado conyugal de la población de 60 + años. Porcentajes. 1970 y 2002.

\begin{tabular}{|l|r|r|r|r|r|r|r|r|}
\hline & \multicolumn{4}{|c|}{ Hombres } & \multicolumn{5}{c|}{ Mujeres } \\
\hline & \multicolumn{3}{|c|}{ Urbano } & \multicolumn{2}{|c|}{ Rural } & \multicolumn{2}{c|}{ Urbano } & \multicolumn{2}{c|}{ Rural } \\
\hline & 1970 & 2002 & 1970 & 2002 & 1970 & 2002 & 1970 & 2002 \\
\hline $\begin{array}{l}\text { Casados/ } \\
\text { Unidos }\end{array}$ & 70.1 & 67.8 & 64.9 & 65.5 & 43.3 & 41.7 & 60.3 & 56.6 \\
\hline Solteros & 16.1 & 10.8 & 23.1 & 13.7 & 13.9 & 9.1 & 7.2 & 6.3 \\
\hline Viudos & 11.0 & 11.5 & 9.8 & 10.2 & 39.0 & 35.1 & 29.8 & 28.4 \\
\hline $\begin{array}{l}\text { Divorcia- } \\
\text { dos }\end{array}$ & 2.8 & 9.9 & 2.2 & 10.6 & 4.7 & 14.1 & 2.7 & 8.7 \\
\hline
\end{tabular}

Fuente: Elaborado a partir de la información de los censos respectivos. 
Es posible que la menor sobre mortalidad masculina, pueda ser la causa de que los cubanos muestren una mayor proporción de viudos. Quedaría por investigar cuál es la causa de la mayor proporción de solteros y solteras uruguayos. En general, las mujeres no presentan diferencias importantes en sus proporciones.

El análisis realizado hasta aquí ha sido de carácter global, dado que aunque intervino el sexo, desde el punto de vista de la edad sólo se utilizaron dos grandes agrupaciones: la población total de 15 años a 29 años y la de 60 años y más. Se hace necesario, con vistas a profundizar el nivel de estudio, la intervención del desglose de las edades.

\section{I.2 El estado conyugal de la población por edades y sexo.}

En el transcurso de la vida de las personas, los hechos demográficos adquieren un gran carácter diferencial, según cambian las agrupaciones que se adopten. Por tanto, a los efectos de verificar los cambios del estado conyugal, en dicho tránsito, resulta interesante hacerlo para cuatro momentos: la juventud, la mediana edad, la tercera y la cuarta edad. Independientemente de que se discute ampliamente sobre cuáles deben ser las edades que comprende cada uno de los momentos del ciclo de vida antes comentado, y se esté o no de acuerdo, en el presente trabajo se adoptó el siguiente esquema:

a) Juventud: 15 a 29 años.

b) Mediana edad: 30 a 59 años.

c) Tercera edad: 60 a 74 años.

d) Cuarta edad: 75 y más años de edad.
Se puede combinar, dentro de cada sexo el estudio de la dinámica del estado conyugal para las personas jóvenes y el de la mediana edad, por sexo.

Es posible que la campaña para legalizar las uniones consensuales, llevada a cabo en los primeros años de la revolución haya sido el factor que determinó el aumento de la proporción de casados del grupo de 15 a 29 años entre 1953 y 1970, tanto en hombres como en mujeres. La influencia de la viudez y las separaciones pueden haber influido en la reducción que muestran estas proporciones entre 1981 y 2002, aunque habría que estudiarlo más detenidamente para poder brindar una conclusión más consistente. En general, la información también indica la influencia de la sobre mortalidad masculina, que se refleja en la mayor viudez femenina.

Debe tenerse presente que los análisis de diferentes censos, por sexos, permiten concluir que por lo general las mujeres declaran mejor su estado conyugal que los hombres, pues ellos en muchas ocasiones ellos tienden a declararse solteros en vez de unidos consensualmente. Esa podría ser una de las razones por las cuales las proporciones de casadas/unidas son muy superiores a las de los hombres en particular en el grupo de 15 a 29 años de edad en los cuatro años en estudio. Pero lo que más tiende a confirmar esta posible explicación es el hecho de que excepto en 1953, en el resto de los censos las proporciones de solteros son muy superiores las de solteras.

Otra hipótesis plausible en cuanto a las razones para que las proporciones masculinas del grupo de 30-59 años

Cuadro 4. Estado conyugal de la población de 60 y +.Países seleccionados. Circa 2000. Porcentajes.

\begin{tabular}{|l|c|c|c|c|c|c|c|c|}
\hline & \multicolumn{4}{|c|}{ Hombres } & \multicolumn{4}{c|}{ Mujeres } \\
\hline & Brasil & Cuba & México & Uruguay & Brasil & Cuba & México & Uruguay \\
\hline & 1992 & 2002 & 2000 & 1996 & 1992 & 2002 & 2000 \\
\hline Casados/Unidos & 80,1 & 67,3 & 77,1 & 72,2 & 41,5 & 44,4 & 45 \\
\hline Solteros & 5,2 & 11,5 & 4,5 & 11,4 & 9,2 & 8,6 & 6,4 \\
\hline Divorciados & 3,8 & 10,1 & 4,2 & 6,2 & 6 & 13,1 & 6,2 \\
\hline Viudos & 10,9 & 11,1 & 14,2 & 10,1 & 43,3 & 33,8 & 42 \\
\hline
\end{tabular}

Fuente: Elaborado a partir de: CEPAL-CELADE (2002): Los adultos mayores en América Latina y el Caribe. Datos e indicadores. Santiago de Chile. 
también sean superiores a las de las féminas es la de la mayor propensión de los hombres a contraer nuevas nupcias una vez roto su vínculo conyugal por cualquier razón.

En general, la comparación de la dinámica del estado conyugal de ambos grupos muestra cómo a medida que aumenta la edad, como es lógico suponer, se incrementa la proporción de casados y unidos, y consecuentemente disminuye la de solteros. Pero el hecho más preocupante del comportamiento de la referida dinámica es el aumento en el tiempo de los porcentajes de divorciados en cada uno de los dos grupos, en particular en el segundo de ellos y con mayor incidencia en el caso de las mujeres, lo cual supone un mayor monto de féminas solas, que están transitando hacia la vejez.

Al confrontar la evolución del estado conyugal de la tercera con la cuarta edad, se aprecia que las proporciones de casadas y unidas del grupo de edades 60-74 refleja, por otra parte, valores muy inferiores para las féminas en los cuatro años mencionados, lo cual puede estar fuertemente asociado con el aumento de la mencionada sobremortalidad masculina, lo cual se verifica al observar que las proporciones de viudas más que triplican los valores reflejados por los hombres.

Uno de los aspectos asociados con los efectos del final de la primera transición demográfica y el desarrollo

\section{Cuadro 5. Cuba: Estado conyugal de la población según sexo y edades seleccionadas.}

1953, 1970, 1981 y 2002. Porcentajes.

\begin{tabular}{|c|c|c|c|c|c|c|c|c|}
\hline & & \multicolumn{3}{|c|}{$15-29$} & \multicolumn{4}{|c|}{$30-59$} \\
\hline Hombres & 1953 & 1970 & 1981 & 2002 & 1953 & 1970 & 1981 & 2002 \\
\hline Casados/Unidos & 23,6 & 34,7 & 31,2 & 31,6 & 76,2 & 81,5 & 80,4 & 72,7 \\
\hline Solteros & 76,1 & 64,0 & 65,2 & 65,6 & 21,3 & 15,4 & 10,5 & 17,4 \\
\hline Divorciados/Separados & 0,2 & 1,2 & 3,5 & 2,7 & 1,5 & 2,6 & 8,3 & 9,3 \\
\hline \multirow[t]{3}{*}{ Viudos } & 0,1 & 0,1 & 0,1 & 0,1 & 1,0 & 0,5 & 0,8 & 0,6 \\
\hline & & \multicolumn{3}{|c|}{$60-74$} & \multicolumn{4}{|c|}{$75+$} \\
\hline & 1953 & 1970 & 1981 & 2002 & 1953 & 1970 & 1981 & 2002 \\
\hline Casados/Unidos & 70,6 & 71,7 & 72,9 & 71,3 & 55 & 56 & 55,8 & 57,5 \\
\hline Solteros & 16,6 & 18,9 & 11,8 & 11,5 & 15,5 & 18 & 15,0 & 11,6 \\
\hline Divorciados/Separados & 0,9 & 2,6 & 8,2 & 11 & 0,7 & 2,4 & 7,3 & 7,7 \\
\hline Viudos & 11,9 & 6,8 & 7,1 & 6,2 & 28,8 & 23,6 & 21,9 & 23,2 \\
\hline \multirow[t]{2}{*}{ Mujeres } & $15-29$ & \multicolumn{3}{|c|}{$30-59$} & & & & \\
\hline & 1953 & 1970 & 1981 & 2002 & 1953 & 1970 & 1981 & 2002 \\
\hline Casadas/Unidas & 46,4 & 56,2 & 49,2 & 49,8 & 76,8 & 82,2 & 77,0 & 71,6 \\
\hline Solteras & 52,4 & 39,5 & 40,9 & 43,5 & 14,2 & 9,7 & 4,2 & 10,1 \\
\hline Divorciados/Separados & 0,9 & 4,0 & 9,7 & 6,5 & 2,5 & 5,8 & 15,1 & 15,8 \\
\hline \multirow[t]{3}{*}{ Viudas } & 0,3 & 0,3 & 0,2 & 0,2 & 6,5 & 2,3 & 3,7 & 2,5 \\
\hline & & \multicolumn{3}{|c|}{$60-74$} & \multicolumn{4}{|c|}{$75+$} \\
\hline & 1953 & 1970 & 1981 & 2002 & 1953 & 1970 & 1981 & 2002 \\
\hline Casadas/Unidas & 47,7 & 53,9 & 53,5 & 53,5 & 22,1 & 26,6 & 23,0 & 25,3 \\
\hline Solteras & 11,2 & 12,7 & 7,8 & 8,3 & 13,0 & 11,1 & 9,6 & 9,4 \\
\hline Divorciados/Separados & 1,5 & 4,7 & 10,9 & 15,5 & 0,6 & 2,6 & 6,7 & 8,1 \\
\hline Viudas & 39,6 & 28,7 & 27,8 & 22,7 & 64,3 & 59,7 & 60,7 & 57,2 \\
\hline
\end{tabular}

Fuente: Elaborado a partir de la información de los censos respectivos. 
de la segunda es el del aumento de los divorcios y Cuba no escapa a esa evolución. Ya el hecho de alcanzar una reducción de la fecundidad, llegando a estar por debajo del nivel del reemplazo desde 1978, puede haber sido uno de los factores asociados con el gran incremento de las proporciones de divorciados para la tercera y cuarta edad, respectivamente, que se reflejan en 1981 y 2002. Por su parte, tanto en un sexo como en otro, el tránsito por los dos grupos mencionados significa un enorme aumento de la viudez, lo cual implica que esto sea considerado por los diseñadores de políticas.

Entre los principales problemas asociados con los adultos mayores está la soledad, al aumentar la pérdida del vínculo conyugal. Por sexo, existen importantes diferencias entre la población adulta mayor, con vínculo y sin vínculo conyugal.

\section{Cuadro 6. Cuba: Población de 60 y + sin vínculo conyugal, según grupos de edades y sexo. 1953, 1981 y 200 . Porcentajes.}

\begin{tabular}{|l|c|c|c|c|c|c|}
\hline & \multicolumn{3}{|c|}{ Hombres } & \multicolumn{3}{c|}{ Mujeres } \\
\hline & 1953 & 1981 & 2002 & 1953 & 1981 & 2002 \\
\hline $60+$ & 32,2 & 31,5 & 32,7 & 57,6 & 54,0 & 55,6 \\
\hline $60-64$ & 25,0 & 25,0 & 26,1 & 45,1 & 38,4 & 41,0 \\
\hline $65-69$ & 29,6 & 26,8 & 29,9 & 54,5 & 47,1 & 46,9 \\
\hline $70-74$ & 34,9 & 31,4 & 31,7 & 65,6 & 51,8 & 54,9 \\
\hline $75+$ & 45,1 & 44,2 & 42,5 & 77,9 & 77,0 & 74,7 \\
\hline
\end{tabular}

Fuente: Elaborado a partir de la información de los censos respectivos.
En general, puede afirmarse que el vivir "sola" es principalmente el destino de las ancianas. Sin embargo, ellas son mejor consideradas entre sus parientes y existe más compromiso hacia ellas, además de ser más independientes en el hogar, sus actividades han estado asociadas con las tareas domésticas y de la vida diaria en el mismo. Independientemente de lo antes expresado, cabe resaltar que la mujer de edad avanzada se halla en una posición mucho más difícil que la de los hombres, lo cual debe tenerse presente por las autoridades competentes. Precisamente, la información que se brinda a continuación ilustra esta situación, pues se aprecia cómo a medida que aumenta la edad, las proporciones de mujeres sin vínculo conyugal se incrementan considerablemente, al punto de que alrededor de tres cuartas partes de ellas ya están solas, hecho también asociado principalmente con la sobremortalidad masculina. También debe tenerse en cuenta que, repetimos, por lo general, los hombres, más que las mujeres, contraen nuevas nupcias al enviudar o separarse de sus cónyuges.

En resumen, con el aumento de la edad, también se eleva el monto de los adultos mayores que ha perdido su pareja y, paralelamente, el vivir de manera solitaria, sin su compañero de toda la vida, lo cual implica un alto costo emocional para ellos y es, a su vez, un hecho social bastante agudo, que afecta tanto su posición en la familia, como en la sociedad y se manifiesta en la disminución de sus funciones en la misma.

Cuadro 7. Población femenina de 60 y + por grupos de edades, sin vínculo conyugal. Países seleccionados. Circa 1990.

\begin{tabular}{|l|c|c|c|c|c|c|c|c|c|c|}
\hline \multicolumn{9}{|c|}{ Hombres } \\
\hline & Brasil 1991 & Chile 1992 & Cuba 1995 & $\begin{array}{c}\text { México } \\
1990\end{array}$ & $\begin{array}{c}\text { Uruguay } \\
1996\end{array}$ & Brasil 1991 & Chile 1992 & $\begin{array}{c}\text { Cuba 1995 } \\
\begin{array}{c}\text { México } \\
1990\end{array}\end{array} \begin{array}{c}\text { Uruguay } \\
1996\end{array}$ \\
\hline $60+$ & 18,0 & 31,7 & 29,8 & 19,5 & 27,8 & 40,9 & 57,4 & 53,7 & 48,9 \\
\hline $60-64$ & 13,0 & 22,0 & 22,6 & 13,3 & 23,5 & 45,1 & 42,7 & 38,2 & 36,1 \\
\hline $65-69$ & 15,4 & 25,2 & 26,2 & 15,7 & 25,0 & 53,3 & 50,8 & 47,0 & 42,8 \\
\hline $70-74$ & 18,8 & 28,9 & 30,1 & 20,0 & 26,9 & 64,1 & 61,2 & 52,4 & 50 \\
\hline $75+$ & 29,2 & 38,6 & 39,0 & 30,3 & 36,3 & 81,4 & 76,6 & 72,6 & 66,2 \\
\hline
\end{tabular}

Fuente: Elaborado a partir de: United Nations: Demographic Yearbook. Special Issue: Historial Supplement, 1948-1997. Para Cuba: CEDEM/IPF/ONE (Centro de Estudios Demográficos/Instituto de Planificación Física/Oficina Nacional de Estadísticas) 1995: Encuesta Nacional de Migración Interna. CEDEM. La Habana. 
Y comparativamente con algunos países de América Latina: ¿la situación es similar o muy desigual? La información que se muestra para la última década del siglo pasado indica que las condiciones no son muy disímiles. En el caso de las mujeres se observan algunas diferencias, en particular para Brasil y México que reportan los más bajos porcentajes sin vínculo conyugal, cuando se trata del grupo de 60 años y más, en tanto que para los otros tres países comparados, al estar en una etapa mucho más avanzada de la transición demográfica, ocurre lo contrario.

La información por edades muestra la difícil situación de las mujeres de 75 años y más, las cuales reflejan enormes proporciones, es decir, por encima del 70 por ciento en 4 de los 5 países. Incluso, en dos de ellos, se arriba y sobrepasa el 80 por ciento.

\section{DINÁMICA DE LA POSICIÓN DE LOS ADULTOS MAYORES EN EL HOGAR.}

"El entorno propicio y favorable para las personas mayores es un tópico destacado del Plan de Acción Internacional de Madrid sobre el Envejecimiento, sin embargo constituye un ámbito de estudio poco explorado en la región. Esto se debe, por una parte, a que abarca un número no menor y heterogéneo de temas $y$, por otra, a que su tratamiento — sobre todo lo referido a los entornos sociales - requiere la producción y análisis de datos cualitativos". ${ }^{2}$

"El concepto de entornos propicios no está plenamente estandarizado, sin embargo el Plan de Acción Internacional de Madrid y su Estrategia Regional aluden especialmente a las condiciones socioculturales y ambientales que propicien un envejecimiento digno y seguro en la comunidad de origen. Estas condiciones se dividen en dos ámbitos de intervención: los entornos sociales y los entornos físicos." ${ }^{3}$

A continuación se presentan diferentes indicadores que muestran la situación de los adultos mayores cubanos, según las propuestas de medición que realizara recientemente el CELADE, en el manual antes citado. No obs- tante, cabe señalar que no se dispone de la información necesaria para realizar un buen análisis de esta parte del trabajo, debido a que no existen encuestas exhaustivas de hogares que profundicen en esta temática.

\section{Cuadro 8. Porcentaje de hogares con personas mayores. Países seleccionados}

\begin{tabular}{|l|c|c|}
\hline Países & 1990 & 2000 \\
\hline Argentina & 34,3 & 34,5 \\
\hline Bolivia & 22,9 & 22,8 \\
\hline Brasil & 21,3 & 22,5 \\
\hline Chile & 29,0 & 29,9 \\
\hline Cuba & -- & 34,2 \\
\hline Ecuador & 23,1 & 27,3 \\
\hline Paraguay & 21,6 & 24,9 \\
\hline Uruguay & 38,4 & 39,9 \\
\hline Rep. Bolivariana de Venezuela & 22,9 & 24,0 \\
\hline
\end{tabular}

Fuente: CELADE (2006): Manual sobre indicadores de calidad de vida en la vejez. Capítulo IV. Envejecimiento y entornos favorables. Santiago de Chile. Pág. 114. ONE (2005): Censo de Población y Viviendas. La Habana.

Según se ha indicado en páginas anteriores, los países que se encuentran en la fase más avanzada de su transición demográfica son los que muestran un mayor grado de envejecimiento de su población. Las proporciones de hogares con personas mayores son muy superiores en dichas naciones, es decir, Argentina, Cuba, Uruguay y en menor medida Chile.

"Además de conocer el número de hogares con personas mayores y el ritmo con el cual se incrementan, es necesario estar al tanto de las modalidades de estructuración familiar que adoptan las personas mayores, dato que tiene una doble importancia: por una parte, determina el perfil de requerimientos habitacionales de este grupo de edad, toda vez que las unidades consumidoras de alojamiento son los hogares, y por otra, el tipo de familia puede tener un peso significativo en sus niveles de bienestar y calidad de vida, situación en la cual pueden ejercer importantes influencias los modelos de política habitacional que implementen los países." ${ }^{4}$ Por tanto, una de las propuestas es la realización de una tipología de hogares con personas mayores según el número de personas en el hogar. 
La tipología elaborada para Cuba, en el caso del año 2002, muestra que en los hogares unipersonales de adultos mayores las diferencias son poco importantes, al igual que los que no cuentan con los mismos. Por otra parte, tanto en zonas rurales como urbanas, los adultos mayores se concentran fundamentalmente en un tamaño de hogares de entre dos y tres personas, con una preponderancia algo mayor en el tipo de hogar rural. En los hogares de entre cuatro y cinco personas, se concentra casi la tercera parte de los adultos mayores y aquí ocurre lo contrario al tamaño anterior: mayor peso en las áreas urbanas. En resumen: los adultos mayores viven fundamentalmente en hogares menores de cinco personas en las áreas urbanas (88\%); algo similar ocurre en las áreas rurales, pero con un menor porcentaje: 82 ; los valores para el total ambas zonas son muy similares. La información anterior es muy útil para los decisores de políticas vinculadas con la vejez.

Según se señaló antes, en realidad es bastante escasa la información sobre esta temática. Esto se demuestra

\section{Cuadro 9. Cuba: Tamaño del hogar con y sin personas mayores,} según área de residencia, 2002. Porcentajes.

\begin{tabular}{|l|c|c|c|c|c|c|}
\hline $\begin{array}{c}\text { Número de } \\
\text { personas }\end{array}$ & $\begin{array}{c}\text { Con } \\
\text { personas }\end{array}$ & $\begin{array}{c}\text { Sin } \\
\text { personas }\end{array}$ & $\begin{array}{c}\text { Con } \\
\text { personas }\end{array}$ & $\begin{array}{c}\text { Sin } \\
\text { personas }\end{array}$ & $\begin{array}{c}\text { Con } \\
\text { personas }\end{array}$ & $\begin{array}{c}\text { Sin } \\
\text { personas }\end{array}$ \\
\hline en el hogar & mayores & mayores & mayores & mayores & mayores & mayores \\
\hline Una & 12,3 & 14,1 & 16,5 & 14,5 & 13,2 & 14,2 \\
\hline Dos a tres & 45,7 & 51,2 & 50,3 & 49,9 & 46,7 & 50,9 \\
\hline $\begin{array}{l}\text { Cuatro a } \\
\text { cinco }\end{array}$ & 30,1 & 30,5 & 24,9 & 31,7 & 28,9 & 30,8 \\
\hline Seis y más & 11,9 & 4,2 & 8,3 & 3,9 & 11,2 & 4,1 \\
\hline Total & 100,0 & 100,0 & 100,0 & 100,0 & 100,0 & 100,0 \\
\hline
\end{tabular}

Fuente: Elaborado a partir de la información de: ONE (2005): Censo de Población y Viviendas. La Habana.

\section{Cuadro 10. Cuba: Distribución porcentual de las personas de $60 \mathrm{y}+$,} según el tamaño del hogar. 2002.

\begin{tabular}{|c|c|c|c|c|c|}
\hline & \multicolumn{5}{|c|}{ Población de 60 y + residentes en hogares con } \\
\hline & Una & Dos a 3 & Cuatro a 5 & Seis $y+$ & $\begin{array}{c}\text { Total de } \\
60+\end{array}$ \\
\hline Hogares de ambas zonas & 9,8 & 48,4 & 29,2 & 12,6 & 100 \\
\hline 1 persona de $60+$ & 19,5 & 42,6 & 28,8 & 9,1 & 100 \\
\hline 2 personas de $60+$ & & 56,9 & 28,3 & 14,8 & 100 \\
\hline 3 personas de $60+$ & & 32,8 & 40,2 & 27 & 100 \\
\hline Hogares urbanos & 9,1 & 46,9 & 30,4 & 13,6 & 100 \\
\hline 1 persona de $60+$ & 18 & 42,6 & 29,8 & 9,6 & 100 \\
\hline 2 personas de $60+$ & & 53,9 & 29,9 & 16,2 & 100 \\
\hline 3 personas de $60+$ & & 30,5 & 40,3 & 29,2 & 100 \\
\hline Hogares rurales & 12 & 53,9 & 25 & 9,1 & 100 \\
\hline 1 persona de $60+$ & 25,2 & 42,5 & 25,1 & 7,2 & 100 \\
\hline 2 personas de $60+$ & & 66,7 & 23,3 & 10 & 100 \\
\hline 3 personas de $60+$ & & 42 & 39,8 & 18,2 & 100 \\
\hline
\end{tabular}

Fuente: Elaborado a partir de la información de: ONE (2005): Censo de Población y Viviendas, 2002 La Habana. por el hecho de que en el propio CELADE, en el manual sobre indicadores de calidad de vida de la vejez, publicado en 2006, sólo presentan información para un país: Nicaragua. Lamentablemente, la tabla que presentan no es comparable con la información cubana, por cuanto llevaron a cabo una distinta distribución según tamaño del hogar.

La antes mencionada tipología puede ampliarse, diferenciando el número de adultos mayores por hogar, que puede ser de uno, dos o tres.

El bajo nivel de la fecundidad cubana, que ha influido en el tamaño de los hogares, por una parte, y por la otra en el rápido proceso de envejecimiento de la población ha determinado que sean en los hogares que cuentan entre dos a tres personas, los que reflejen en la zona rural los mayores porcentajes de 2 adultos mayores que residen en los mismos, con un valor muy superior al correspondiente a las áreas urbanas. Sin embargo, 
debe tenerse presente que sólo el $21.9 \%$ de los adultos mayores reside en las zonas rurales. Además, que el porcentaje de hogares rurales en que residen adultos mayores es sólo de 21.6. Por otra parte, sí resulta de interés conocer que la proporción de adultos mayores por hogar es mayor en las zonas rurales que en las urbanas, es decir, 1.37 contra 1.34. Estos valores constituyen una primera aproximación para conocer la estructura familiar.

Tal como se indicó en páginas anteriores, con el proceso de envejecimiento ocurren cambios en la composición de la familia. Más específicamente, se van produciendo mutaciones en la posición de los adultos mayores dentro del hogar. A continuación se realiza un breve análisis de estos aspectos, según el sexo, la edad y el lugar de residencia de las personas mayores en el caso cubano, para lo cual se han usado, en unos casos sólo 2 censos en aras de evitar un gran monto de información. En otros, se han empleado tres censos.

Según se aprecia en la información citada, en los últimos 32 años, el rol de los adultos mayores como jefes del hogar se ha visto reducido en el caso de los hombres y, concomitantemente, ha aumentado el de las mujeres, como un reflejo del citado proceso de envejecimiento de la población cubana. La causa principal de este cambio está asociada con la sobremortalidad masculina. Sin embargo, cabe señalar que las proporciones de hombres se acercan algo al doble en cuanto a su papel de jefes, en comparación con el de las mujeres.
Cuando el análisis se realiza según áreas de residencia y sexo, se nota la incidencia de una situación inferior de las mujeres en las zonas rurales, pues, por ejemplo, en 1981, la proporción de jefas era un poco más de la mitad de la reflejada en la zona urbana, en tanto en 2002 fue de dos tercios. Pero lo más notable, sin embargo, es el enorme valor de las proporciones de cónyuges, en el caso de las mujeres, que incluso superan con creces las de jefas. Este comportamiento está asociado con la mayor mortalidad de estas zonas. El censo de 1970 no brindó información por áreas de residencia.
Cuadro 11. Cuba: Distribución porcentual del tamaño del hogar en que residen las personas de $60 \mathrm{y}+.2002$.

\begin{tabular}{|l|c|c|c|c|c|}
\hline & \multicolumn{5}{|c|}{ Población de $\mathbf{6 0} \mathbf{y}+$ residentes en hogares } \\
\cline { 2 - 6 } & Una & Dos a 3 & Cuatro a 5 & Seis y + & Total de 60+ \\
\hline Hogares de ambas zonas & 100,0 & 100,0 & 100,0 & 100,0 & 100,0 \\
\hline 1 persona de 60+ & 100,0 & 44,1 & 49,4 & 36,1 & 50,1 \\
\hline 2 personas de 60+ & & 52,3 & 43,2 & 52,3 & 44,5 \\
\hline 3 personas de 60+ & & 3,6 & 7,4 & 11,6 & 5,4 \\
\hline Hogares urbanos & 100,0 & 100,0 & 100,0 & 100,0 & 100,0 \\
\hline 1 persona de 60+ & 100,0 & 46,1 & 49,7 & 35,8 & 50,7 \\
\hline 2 personas de 60+ & & 50,3 & 43,0 & 52,3 & 43,8 \\
\hline 3 personas de 60+ & & 3,6 & 7,3 & 11,9 & 5,5 \\
\hline Hogares rurales & 100,0 & 100,0 & 100,0 & 100,0 & 100,0 \\
\hline 1 persona de 60+ 60 & 100,0 & 37,7 & 48,1 & 38,1 & 47,8 \\
\hline 2 personas de 60+ & & 58,4 & 44,0 & 51,8 & 47,2 \\
\hline 3 personas de 60+ & & 3,9 & 7,9 & 10,1 & 5,0 \\
\hline
\end{tabular}

Fuente: Elaborado a partir de la información de: ONE (2005): Censo de Población y Viviendas. La Habana.

\section{Cuadro 12. Cuba: Población de $60 \mathrm{y}+\mathrm{y}$ su relación con el jefe del hogar. Porcentajes. 1970, 1981 y 2002.}

\begin{tabular}{|l|r|r|r|r|r|r|}
\hline \multirow{2}{*}{ Jefe } & \multicolumn{3}{|c|}{ Hombres } & \multicolumn{3}{|c|}{ Mujeres } \\
\cline { 2 - 7 } & 1970 & 1981 & 2002 & 1970 & 1981 & 2002 \\
\hline Cónyuge & 80,4 & 80,4 & 75,8 & 30,4 & 38,9 & 48,5 \\
\hline Pariente del jefe & 1,6 & 4,3 & 9,6 & 35,4 & 37,6 & 32,4 \\
\hline Otros parientes & 7,9 & 6,7 & 7,2 & 23,6 & 15,2 & 12,7 \\
\hline Otros no parientes & 5,8 & 5,4 & 5,0 & 8,6 & 6,4 & 4,9 \\
\hline Miembros de colectividades & 2,5 & 2,0 & 1,4 & 1,2 & 1,3 & 0,9 \\
\hline
\end{tabular}

Fuente: Elaborado a partir de la información de los censos respectivos. 
El análisis anterior se torna más interesante si el mismo se lleva a cabo por grupos de edades. Comencemos con el año de 1981. Posiblemente la sobremortalidad masculina, por una parte y la mayor propensión de los adultos mayores a contraer nuevas nupcias, una vez que no poseen vínculo conyugal, sea la causa del aumento del porcentaje de cónyuges en el caso de los hombres y su disminución en el caso de las mujeres. También cabe tener presente que al
Cuadro 13. Cuba: Población de 60 y + y su relación con el jefe del hogar, según zonas de residencia. Porcentajes. 1981 y 2002.

\begin{tabular}{|l|r|r|r|r|r|r|r|r|r|}
\hline \multirow{2}{*}{} & \multicolumn{4}{|c|}{ Hombres } & \multicolumn{4}{c|}{ Mujeres } \\
\cline { 2 - 12 } & \multicolumn{2}{|c|}{ Urbano } & \multicolumn{2}{|c|}{ Rural } & \multicolumn{2}{|c|}{ Urbano } & \multicolumn{2}{c|}{ Rural } \\
\hline & 1981 & 2002 & 1981 & 2002 & 1981 & 2002 & 1981 & 2002 \\
\hline Jefe & 78,4 & 73,3 & 84,7 & 83,0 & 43,1 & 51,4 & 23,1 & 34,8 \\
\hline Cónyuge & 5,8 & 11,5 & 1,1 & 4,1 & 33,1 & 28,8 & 54,5 & 48,6 \\
\hline Pariente del jefe & 6,9 & 7,4 & 6,3 & 6,9 & 14,9 & 13,0 & 16,4 & 12,5 \\
\hline Otros parientes & 5,5 & 5,2 & 5,3 & 4,5 & 6,8 & 5,2 & 5,0 & 3,4 \\
\hline Otros no parientes & 1,9 & 1,5 & 2,1 & 1,4 & 1,4 & 0,9 & 0,9 & 0,6 \\
\hline Miembros de colectividades & 1,5 & 1,2 & 0,4 & 0,5 & 0,8 & 0,7 & 0,1 & 0,2 \\
\hline
\end{tabular}

Fuente: Elaborado a partir de la información de los censos respectivos. ser mucho menor su rol de jefes, sea más lógico que muestren un mayor peso como cónyuges, e incluso como parientas del jefe. En el caso de las mujeres, al ser menor su rol como jefas, resulta lógico que la estructura sea totalmente distinta.

Al comparar la posición de los adultos mayores en el hogar de 1970 con la de 1981 y 2002 , surgen algunos aspectos interesantes, los cuales requieren

Cuadro 14. Cuba: Población de 60 y + su relación con el jefe del hogar. Porcentajes. 1970, 1981 y 2002.

\begin{tabular}{|c|c|c|c|c|c|c|c|c|c|c|c|c|}
\hline & \multicolumn{6}{|c|}{ Hombres } & \multicolumn{6}{|c|}{ Mujeres } \\
\hline 1970 & $60-64$ & $65-69$ & $70-74$ & $75-79$ & $80-84$ & $85+$ & $60-64$ & $65-69$ & $70-74$ & $75-79$ & $80-84$ & $85+$ \\
\hline Jefe & 86,4 & 84,2 & 79,0 & 74,5 & 66,6 & 52,5 & 27,6 & 32,2 & 35,0 & 34,0 & 31,0 & 22,8 \\
\hline Cónyuge & 1,6 & 1,6 & 1,6 & 1,7 & 1,5 & 1,3 & 51,4 & 39,2 & 27,5 & 20,0 & 11,9 & 5,9 \\
\hline Pariente del jefe & 3,5 & 4,8 & 7,6 & 12,4 & 19,4 & 31,2 & 12,7 & 19,1 & 25,9 & 33,9 & 42,8 & 54,1 \\
\hline Otros parientes & 5,8 & 5,6 & 5,9 & 5,9 & 6,1 & 7,3 & 7,1 & 7,9 & 9,3 & 9,5 & 11,0 & 13,1 \\
\hline Otros no parientes & 1,6 & 2,5 & 3,6 & 3,1 & 3,1 & 3,3 & 0,8 & 1,1 & 1,3 & 1,5 & 1,7 & 1,9 \\
\hline Miembros colectivid & 1,1 & 1,3 & 2,3 & 2,4 & 3,3 & 4,4 & 0,4 & 0,5 & 1,0 & 1,1 & 1,6 & 2,2 \\
\hline \multicolumn{13}{|l|}{1981} \\
\hline Jefe & 83,7 & 83,3 & 81,4 & 77,9 & 71,0 & 58,8 & 34,4 & 38,2 & 41,8 & 44,5 & 44,1 & 36,0 \\
\hline Cónyuge & 5,1 & 4,6 & 4,0 & 3,4 & 3,3 & 2,5 & 51,8 & 44,0 & 34,1 & 22,3 & 12,2 & 5,4 \\
\hline Pariente del jefe & 3,6 & 4,3 & 6,3 & 9,3 & 13,3 & 24,5 & 7,9 & 10,7 & 15,4 & 22,7 & 30,2 & 42,7 \\
\hline Otros parientes & 5,4 & 5,4 & 5,5 & 5,3 & 5,5 & 6,1 & 4,7 & 5,6 & 6,9 & 8,0 & 9,4 & 10,3 \\
\hline Otros no parientes & 1,5 & 1,7 & 1,8 & 2,4 & 3,6 & 3,4 & 1,0 & 1,1 & 1,2 & 1,6 & 2,3 & 2,9 \\
\hline Miembros colectivid & 0,6 & 0,6 & 0,9 & 1,7 & 3,4 & 4,7 & 0,2 & 0,3 & 0,5 & 0,9 & 1,8 & 2,7 \\
\hline \multicolumn{13}{|l|}{2002} \\
\hline Jefe & 76,7 & 79,1 & 78,9 & 76,4 & 71,2 & 58,6 & 47,2 & 49,2 & 51,1 & 51,6 & 48,1 & 39,9 \\
\hline Cónyuge & 12,3 & 10,3 & 9,1 & 7,6 & 6,3 & 4,7 & 43,4 & 39,6 & 33,4 & 24,7 & 16,0 & 6,8 \\
\hline Pariente del jefe & 4,9 & 4,3 & 5,1 & 7,8 & 12,8 & 24,2 & 4,8 & 7,0 & 10,2 & 16,2 & 24,9 & 37,5 \\
\hline Otros parientes & 4,4 & 4,5 & 4,7 & 5,4 & 6,2 & 7,3 & 2,6 & 3,2 & 4,3 & 6,0 & 8,6 & 12,1 \\
\hline Otros no parientes & 1,4 & 1,3 & 1,3 & 1,5 & 1,7 & 2,1 & 0,7 & 0,6 & 0,7 & 0,9 & 1,4 & 2,1 \\
\hline Miembros colectivid & 0,4 & 0,6 & 0,9 & 1,4 & 1,9 & 3,2 & 0,3 & 0,4 & 0,4 & 0,7 & 1,1 & 1,7 \\
\hline
\end{tabular}

Fuente: Elaborado a partir de la información de los censos respectivos. 
de un mayor análisis del que se realizará a continuación. Al producirse en dicho intervalos de tiempo un aumento de la intensidad del proceso de envejecimiento de la población cubana, asociado con la gran reducción de la fecundidad, que desde 1978 se ubicó por debajo del nivel de reemplazo de la población, se nota una pequeña reducción de las proporciones de jefes masculinos y un mayor aumento de las jefas, las cuales también reflejan un aumento como cónyuges, al igual que los hombres, según se indicó con anterioridad. Por otra parte, en ambos años, al reducirse las proporciones de jefes y jefas, así como de cónyuges, en el grupo de 85 años y más, se "dispara” la proporción de Parientes del Jefe en los dos sexos.

La composición del vínculo conyugal de los adultos mayores no hace más que confirmar lo que se había indicado antes: Con el paso del tiempo el peso relativo de los hombres como jefes con vínculo conyugal disminuye, como cabría esperar, con el concomitante aumento en el caso de las mujeres. Asimismo, aumenta la importancia de los otros parientes y no parientes con vínculo conyugal. Se reitera lo señalado antes: esta es una de las cuestiones menos estudiadas y por tanto se hace necesario una mayor investigación sobre esta temática.

Independientemente de que se brindó información al respecto, se podría hacer el análisis de la dinámica solo de las tasas de jefatura, por sexos y edades, a los efectos de verificar de manera particular, cuál es el grado de veracidad de los planteamientos que se han hecho en párrafos anteriores.

\section{Cuadro 15. Cuba: Vínculo conyugal de la población de 60 y +, según su relación con el jefe del hogar. 1981 y 2002.}

\begin{tabular}{|l|c|c|c|c|c|c|c|c|}
\hline \multirow{2}{*}{} & \multicolumn{4}{|c|}{ Con vínculo conyugal } & \multicolumn{3}{c|}{ Sin vínculo conyugal } \\
\cline { 2 - 12 } & \multicolumn{2}{|c|}{1981} & \multicolumn{2}{c|}{2002} & \multicolumn{2}{c|}{1981} & \multicolumn{2}{c|}{2002} \\
\hline Hom- & Hom- & $\begin{array}{c}\text { Hom- } \\
\text { bres }\end{array}$ & Mujeres & Mujeres & $\begin{array}{c}\text { Hom- } \\
\text { bres }\end{array}$ & Mujeres & $\begin{array}{c}\text { Hom- } \\
\text { bres }\end{array}$ & Mujeres \\
\hline Jefe & 75,4 & 12,9 & 71,3 & 19,0 & 24,6 & 87,1 & 28,7 & 81,0 \\
\hline Esposa & 10,0 & 100,0 & 100,0 & 100,0 & 100,0 & 100,0 & 100,0 & 100,0 \\
\hline Parientes del jefe & 38,4 & 17,2 & 34,1 & 15,8 & 61,6 & 82,8 & 65,9 & 84,2 \\
\hline Otros parientes & 11,5 & 8,8 & 14,8 & 11,7 & 88,5 & 91,2 & 85,2 & 88,3 \\
\hline Otros no parientes & 15,9 & 12,5 & 21,3 & 14,2 & 84,1 & 87,5 & 78,7 & 85,8 \\
\hline
\end{tabular}

Fuente: Elaborado a partir de la información de los censos respectivos.
Cuadro 16. Cuba: Tasas de jefatura de los adultos mayores, según sexo y edad. 1970, 1981 y 2002.

\begin{tabular}{|l|l|l|l|l|l|l|}
\hline & \multicolumn{3}{|c|}{ Hombres } & \multicolumn{3}{c|}{ Mujeres } \\
\hline & 1970 & 1981 & 2002 & 1970 & 1981 & 2002 \\
\hline $60+$ & 80,4 & 81,4 & 76,6 & 30,4 & 39,1 & 48,6 \\
\hline $60-64$ & 86,3 & 84,2 & 77,0 & 27,6 & 34,5 & 47,3 \\
\hline $65-69$ & 84,3 & 83,9 & 79,5 & 32,2 & 38,3 & 49,4 \\
\hline $70-74$ & 79,1 & 82,5 & 79,6 & 35,0 & 42,1 & 51,3 \\
\hline $75-79$ & 74,5 & 79,2 & 77,4 & 34,0 & 44,9 & 52,0 \\
\hline $80-84$ & 66,6 & 71,0 & 72,6 & 31,0 & 44,1 & 48,6 \\
\hline $85+$ & 52,5 & 61,6 & 60,5 & 22,8 & 37,0 & 40,6 \\
\hline
\end{tabular}

Fuente: Elaborado a partir de la información de los censos respectivos.

Durante el período 1970-2002, las tasas de jefatura masculinas han experimentado un comportamiento bastante diferencial por edades. En efecto, el grupo 60 69 años de edad cambios ínfimos, en tanto el de 70-74 cambió muy poco. Sin embargo, en comparación con 1970, las tasas de los años 1981 y 2002, las tasas de los tres grupos siguientes fueron superiores, lo cual se contradice con lo que cabría esperar.

Por su parte, las tasas femeninas si muestran más claramente el aumento del papel de la mujer en el hogar, pues las mismas aumentan sistemáticamente para todas las edades durante el período analizado.

Cabría preguntarse cuál fue el comportamiento de dichas tasas según la zona residencia. El siguiente gráfico brinda dicha información para 1981 y 2002, debido a que el Censo de 1970 no brindó tal desglose.

En la zona urbana, la disminución mayor la reporta el grupo de 60-64 años de edad, en el caso de los hombres, mientras que el resto de las edades muestran decrecimientos mucho menores. Sin embargo, los hombres rurales son los que marcan la pauta similar a la del total de ambas zonas, es decir, disminución de los valores 


\section{Cuadro 17. Cuba: Tasas de jefatura de los adultos mayores según zonas de residencia. Porcentajes. 1981 y 2002.}

\begin{tabular}{|l|r|r|r|r|r|r|r|r|}
\hline \multirow{2}{*}{} & \multicolumn{4}{|c|}{ Zona urbana } & \multicolumn{4}{c|}{ Zona rural } \\
\cline { 2 - 10 } & \multicolumn{2}{|c|}{ Hombres } & \multicolumn{2}{|c|}{ Mujeres } & \multicolumn{2}{|c|}{ Hombres } & \multicolumn{2}{c|}{ Mujeres } \\
\hline & 1981 & 2002 & 1981 & 2002 & 1981 & 2002 & 1981 & 2002 \\
\hline $60+$ & 79,7 & 74,3 & 43,4 & 51,7 & 85,1 & 83,0 & 23,1 & 34,8 \\
\hline $60-64$ & 81,9 & 74,3 & 39,1 & 51,2 & 89,2 & 85,0 & 19,0 & 31,0 \\
\hline $65-69$ & 81,4 & 77,1 & 42,8 & 53,2 & 88,0 & 86,5 & 22,1 & 33,5 \\
\hline $70-74$ & 79,7 & 77,3 & 46,2 & 54,4 & 85,7 & 86,0 & 26,0 & 37,2 \\
\hline $75-79$ & 77,5 & 75,5 & 48,8 & 54,4 & 81,3 & 83,0 & 28,7 & 40,2 \\
\hline $80-84$ & 72,2 & 70,9 & 48,3 & 50,5 & 76,2 & 77,4 & 29,2 & 39,0 \\
\hline $85+$ & 57,6 & 59,0 & 40,1 & 41,7 & 62,2 & 64,6 & 24,1 & 34,9 \\
\hline
\end{tabular}

Fuente: Elaborado a partir de la información de los censos respectivos.

en los dos primeros grupos de edades y aumento de los mismos en el resto.

Las mujeres de ambas zonas sí muestran un comportamiento más uniforme, en este caso, aumento de sus tasas, en algunos casos de más de 10 puntos porcentuales. También, queda reflejado el avance de la mujer, debido a que sus tasas se incrementan en alrededor de 12 puntos porcentuales, tanto en las zonas rurales como en las urbanas. No obstante, cabe esperar que el mayor desarrollo social la población, determine que las tasas de las mujeres de las zonas rurales lleguen a aproximarse sensiblemente a las de las mujeres de las zonas urbanas, o quizás las igualen.

\section{REFLEXIONES FINALES}

Indudablemente, el proceso de envejecimiento repercutió sobre el estado conyugal de la población cubana en la segunda mitad del siglo pasado. El relativamente conciso estudio realizado en cuanto al citado estado conyugal de los adultos mayores, así como las variaciones en la relación de ellos con el jefe del hogar, llaman a un verdadero estudio interdisciplinario, es decir, la participación de diferentes especialistas, demógrafos, sicólogos, sociólogos, etc., que contribuyan a delimitar más claramente cuáles son los factores que están incidiendo en los distintos comportamientos, así como cuáles pueden ser las futuras implicaciones de todo tipo. En definitiva, este trabajo se propuso alertar acerca de la complejidad de este objeto de estudio, pues son cientos de miles las personas de diferente sexo, lugar de residencia, grupo de edad, estado conyugal, nivel de participación en la actividad económica las que claman por sacar a la luz pública toda esta problemática y su dinámica. No menos importante es la participación de los formuladores de políticas, los que toman las decisiones a diferentes niveles y las instituciones del estado en cuanto a la toma de conciencia de la importancia del problema.

Además de las consideraciones anteriores, se pudo constar cómo el papel de la mujer dentro del hogar ha mejorado considerablemente, como resultado de las transformaciones de la revolución, lo cual se reflejó en el aumento sistemático de sus tasas de jefatura. 
${ }^{1}$ CEPAL-CELADE (2002): Los adultos mayores en América Latina y el Caribe.

Datos e indicadores. Santiago de Chile.

${ }^{2}$ CELADE (2006): Manual sobre indicadores de calidad de vida en la vejez.

Capítulo IV. Envejecimiento y entornos favorables. Santiago de Chile. Pág. 113.

${ }^{3}$ IBIDEM.

${ }^{4}$ IBIDEM.

\section{BIBLIOGRAFÍA}

CEE (Comité Estatal de Estadísticas) 1984: Censo de Población y Viviendas, 1981. República de Cuba. Oficina Nacional del Censo. La Habana.

CEDEM/IPF/ONE (Centro de Estudios Demográficos/Instituto de Planificación Física/Oficina Nacional de Estadísticas) (1995):Encuesta Nacional de Migración Interna. CEDEM. La Habana.

CELADE (2006): Manual sobre indicadores de calidad de vida en la vejez. Santiago de Chile.

CEPAL-CELADE (2002): Los adultos mayores en América

Latina y el Caribe. Datos e indicadores. Santiago de Chile.

United Nations: Demographic Yearbook. Special Issue:

Historial Supplement, 1948-1997. New Cork.

Oficina Nacional de Estadísticas (ONE) (2006): Censo de

Población y Viviendas. Cuba. 2002. La Habana.

ONE (2004): El envejecimiento de la población cubana. Cifras para su estudio. La Habana

TRIBUNAL SUPERIOR ELECTORAL, 1955: República de Cuba. Censo de Población, Viviendas y Electoral. 1953. Informe General. Oficina Nacional de los Censos Demográfico y Electoral. P. Fernández y Cía., S. en C. La Habana.

United Nations (2001): World Population Prospects. The 2000 Revision. Volume II: Sex and Age.

United Nations 1956: The aging of Populations and its Economic and Social Implications. Estudios de población, no 26 ( $\mathrm{n}^{\mathrm{o}}$ de venta 1956.6), y Population Ageing 1999 (n⿳亠丷厂 de venta E.99.

XIII.II). 